\title{
Search for EWK production of SUSY in final states with multiple leptons at the CMS experiment at the CERN LHC
}

\author{
Constantin Heidegger* \\ on behalf of the CMS Collaboration \\ ETH Zurich, Institute for Particle Physics and Astrophysics, \\ Otto-Stern-Weg 5, 8093 Zurich, Switzerland \\ E-mail: constantin.heideggerecern.ch
}

\begin{abstract}
This poster reports on the search for the production of charginos and neutralinos in events with either two leptons of the same charge or three or more leptons using the full 2016 proton-proton collision dataset of $35.9 \mathrm{fb}^{-1}$ at $\sqrt{s}=13 \mathrm{TeV}$ collected by the CMS detector. Exclusion limits at $95 \%$ confidence level range between $450-1100 \mathrm{GeV}$ depending on the SUSY scenario.
\end{abstract}

EPS-HEP 2017, European Physical Society conference on High Energy Physics 5-12 July 2017

Venice, Italy

\footnotetext{
* Speaker.
} 


\section{Introduction}

Supersymmetry (SUSY) is able to solve a number of questions the standard model of particle physics (SM) has left unresolved. Particle collision experiments at the CERN LHC such as CMS [1], perform searches for evidence for SUSY in proton-proton collisions in a variety of different final states. The analysis presented in this poster has been reported in Ref. [2] and focusses on the production of wino-like chargino-neutralino $\left(\widetilde{\chi}_{1}^{ \pm} \widetilde{\chi}_{2}^{0}\right)$ production in final states with either two leptons of the same charge or three or more leptons. Charginos and neutralinos are assumed mass degenerate, $m_{\widetilde{\chi}_{1}^{ \pm}}=m_{\widetilde{\chi}_{2}^{0}}$, and the bino-like $\widetilde{\chi}_{1}^{0}$ is the lightest supersymmetric particle (LSP)

\section{Signal topology}

Example simplified models of $\widetilde{\chi}_{1}^{ \pm} \widetilde{\chi}_{2}^{0}$ production are shown in Figure 1. Two major scenarios in terms of the masses of the $\tilde{\ell}$, the SUSY partners of the SM leptons (charged leptons $(\ell=\mathrm{e}, \mu$, $\tau)$ and neutrinos), are considered: in the case of light $\tilde{\ell}$, these particles serve as mediators of the decay, while in the case of heavy $\tilde{\ell}$ the $\widetilde{\chi}_{1}^{ \pm} \widetilde{\chi}_{2}^{0}$ pair decays via vector bosons. The light $\tilde{\ell}$ scenario can be subdivided by imposing an assumption on the flavor of the $\widetilde{\ell}$ involved in the decay: $\widetilde{\chi}_{1}^{ \pm}$and $\widetilde{\chi}_{2}^{0}$ can decay to all three flavors with equal probability (flavor-democratic scenario, Fig. 1 (left)), or the decay to the $\tau$ lepton flavor is preferred (tau-dominated scenario, Fig. 1 (middle)). The $\tau$ lepton can decay to lighter leptons (e, $\mu$ ) or it can decay hadronically in which case it is referred to as $\tau_{\mathrm{h}}$. The heavy $\tilde{\ell}$ scenario is shown in Figure 1 (right). In total, 12 different SUSY scenarios have been studied.
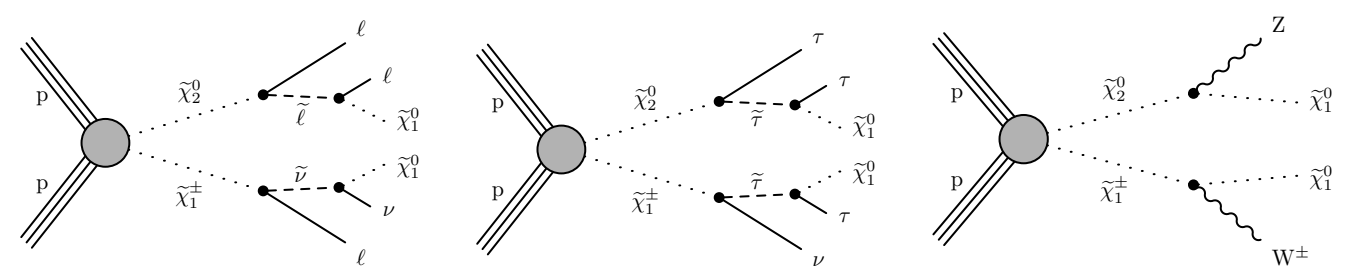

Figure 1: Example processes of $\widetilde{\chi}_{1}^{ \pm} \widetilde{\chi}_{2}^{0}$ production with three different decay modes: light slepton scenario with flavor-democratic decays (left), light slepton scenario with tau-dominated decays (middle), and heavy slepton scenario with decays via vector bosons. All topologies yield three leptons in the final state and little hadronic activity. Figures taken from [2].

All decay modes yield at least three $\ell$ in the final state and one also can expect a significant amount of missing transverse energy $\left(p_{\mathrm{T}}^{\text {miss }}\right.$ ) given the presence of the two $\widetilde{\chi}_{1}^{0}$ and the $v$. In order to reduce contributions from processes such as $\mathrm{tt} V$, events with at least one b-tagged jet are rejected. The selected events are categorized according to the number of $\ell$, the lepton flavor, the presence of pairs of $\ell$ with opposite sign (OS), and the presence of OS pairs with same flavor (OSSF). Within each of the 12 event categories, a finer granularity binning is done according to a suitable set of kinematic variables aiming to separate signal and background processes for different model topologies. 


\section{Lepton identification}

An important distinction is to be made between $\ell$ that have been produced at the primary vertex (i.e. coming from a decay of a $\mathrm{W}, \mathrm{Z}$ or $\mathrm{H}$ boson or from a SUSY particle decay) and $\ell$ that have been produced elsewhere. The former is called "prompt" (or "signal-like"), the latter is referred to as "nonprompt" (or "background-like"). The source of nonprompt leptons depends on their flavor. For e and $\tau$ leptons, nonprompt leptons typically are misreconstructed light flavor jets (with pions or Kaons as main constituent). Nonprompt $\mu$ mostly are real $\mu$ produced in the decay of a b hadron.

In order to separate prompt from nonprompt $\ell$, a multi-variate lepton identification method (MVA) is deployed - a boosted decision tree (BDT) trained to accept prompt and reject nonprompt leptons. Impact parameter variables and observables related to the jet that is closest to the $\ell$ serve as input to the BDT. Figure 2 (left) shows the MVA score on the trailing lepton in a trilepton selection. It demonstrates the power of the MVA to separate the Drell-Yan $(\mathrm{Z}+\mathrm{X})$ processes, where the third lepton is nonprompt given that it originates from the jet $(\mathrm{X})$, from the $\mathrm{VZ}$ process which contains at least three prompt leptons in the final state. The BDT improves the background rejection at the same signal acceptance with respect to a conventional "cut-based" lepton selection. This results in a better sensitivity of the analysis of approximately $10 \%$.
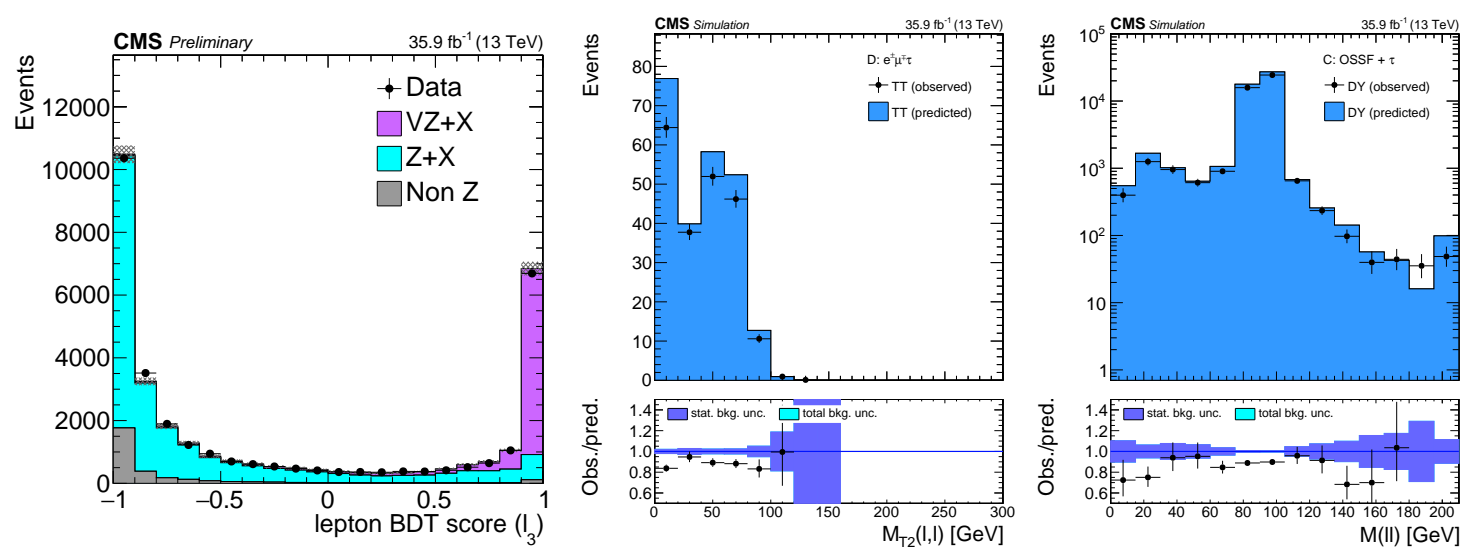

Figure 2: Left: MVA output (score) for the third lepton in events with at least two prompt leptons. The MVA nicely separates prompt leptons $(\mathrm{VZ}+\mathrm{X})$ from nonprompt leptons $(\mathrm{Z}+\mathrm{X}$, Non-Z). Middle and right: closure test of the tight-to-loose ratio in events with two e or $\mu$ of opposite charge (middle) and same flavor (right) and one $\tau_{\mathrm{h}}$ : the data points show the distribution of $M_{\mathrm{T} 2}$ (middle) and $M_{\ell \ell}$ (right) for simulated events containing at least one nonprompt lepton while the filled histogram shows the prediction of these events using the tight-to-loose method. Figures taken from [2].

\section{Residual nonprompt background}

Since no lepton identification is perfect, there is a residual nonprompt contamination in the search regions. This residual nonprompt background is estimated with a data-driven method which, in simple words, can be described as follows: measure the probability $p_{T L}$ for a nonprompt $\ell$ to pass the full lepton selection given that it has already passed a looser version. This probability $p_{T L}$ is called the "tight-loose ratio" and it is used to predict the background yields in the search 
regions. The data control sample where $p_{T L}$ is measured is called the "measurement region", and it is enriched in nonprompt $\ell$ by selecting dijet events where one of the jets is reconstructed as a $\ell$. The tight-loose ratio $p_{T L}$ is of order 5-20\% depending on the lepton- $p_{T}$, the lepton- $|\eta|$ (absolute value of the lepton pseudorapidity), and the lepton flavor ( $p_{T L}$ is largest for $\tau$ 's).

The predictive power of the method is validated in a so-called "closure test" comparing true yields in the search region from simulated events with nonprompt leptons (using generator truth information) to the yields obtained from the data-driven method. Figure 2 shows two examples of a closure test in events with a pair of e or $\mu$ of opposite charge and one $\tau_{\mathrm{h}}$ (middle) and in events with an OSSF pair of e or $\mu$ and one $\tau_{\mathrm{h}}$ (right). The number of simulated events with at least one nonprompt lepton is compared to the prediction from the tight-to-loose method. From the result of closure tests such as these, a systematic uncertainty of $30 \%$ is attributed to the method.

\section{Prompt background}

The prompt SM background is constituted of SM processes that contain at least three prompt $\ell$ and $p_{\mathrm{T}}^{\text {miss }}$ in the final state. Given their similarity to the signal, they are particularly challenging to reduce. The process with the largest contribution is SM WZ production. For this background, one estimates the shape of the kinematic observables used in the event categorization $\left(M_{\ell \ell}, M_{\mathrm{T}}\right.$, and $p_{\mathrm{T}}^{\text {miss }}$ ) from simulated events while the overall normalization is assessed in a data control sample. Two additional control regions are used to verify the modeling of the $p_{\mathrm{T}}^{\text {miss }}$ and $M_{\mathrm{T}}$ distributions. These observables are crucial as any SUSY signal is expected to appear at large values while SM WZ will peak at lower values.
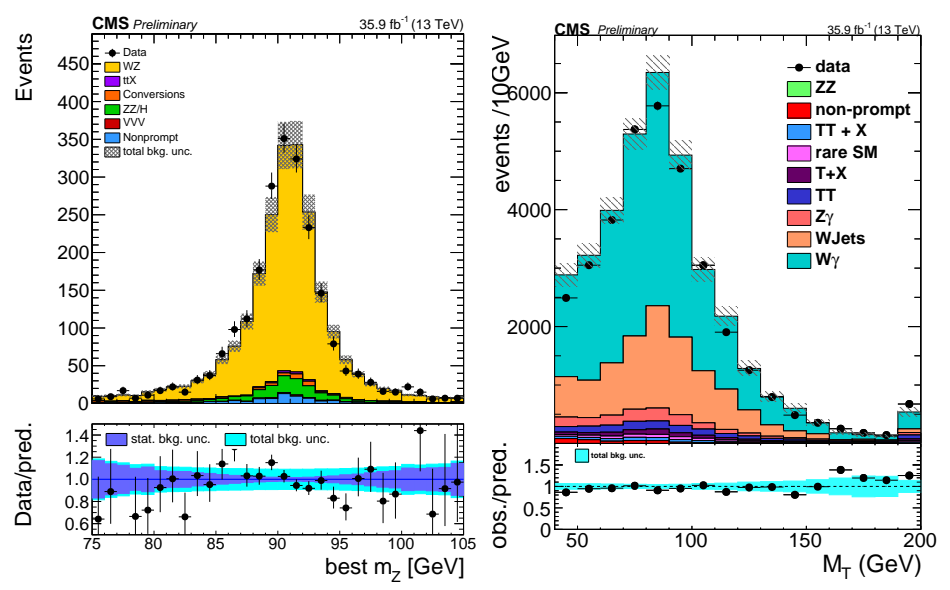

Figure 3: Left: distribution of $M_{\ell \ell}$ of the OSSF pair in the data control sample enriched in SM WZ production. Right: distribution of $M_{\mathrm{T}}$ in a dedicated control region used to verify the modeling of $M_{\mathrm{T}}$ and $p_{\mathrm{T}}^{\text {miss }}$. Figures taken from [2].

In simple words, the ultimate challenge of this analysis is to find a shape difference between signal and prompt SM background processes such as WZ in the kinematic observables relevant in each of the 12 event categories. 


\section{Results and interpretations}

In Figure 4, the results for two event categories are presented: the data-to-prediction comparison in the 44 search regions for events with three e or $\mu$ and an OSSF pair is presented on the left. The binning is done according to the $M_{\ell \ell}$ of the OSSF pair, the $M_{\mathrm{T}}$ of the third lepton, and $p_{\mathrm{T}}^{\text {miss }}$. Figure 4 (right) shows the distribution of $M_{\mathrm{T} 2}(\ell, \ell)$ in events with an OSSF pair of e and $\mu$, and one $\tau_{\mathrm{h}}$. While the signal is expected to appear in regions of either high $p_{\mathrm{T}}^{\text {miss }}$ or $M_{\mathrm{T} 2}$, no significant excess over the SM prediction has been observed.
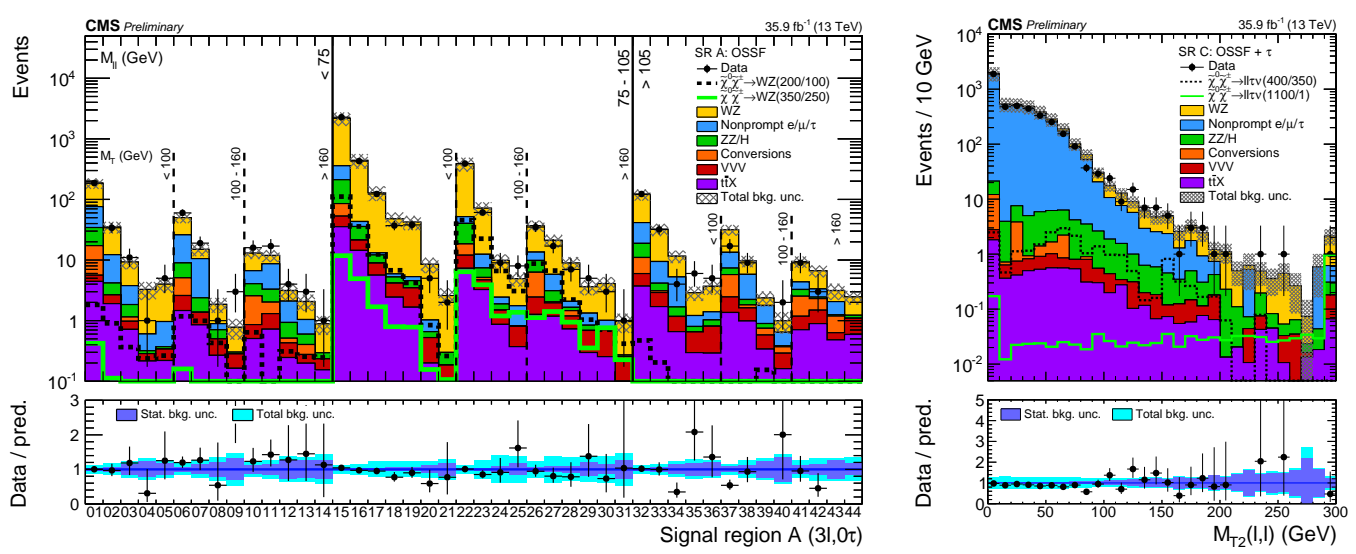

Figure 4: Data-to-prediction comparison in the search regions for events with three e or $\mu$ and an OSSF pair (left) and as a function of $M_{\mathrm{T} 2}$ for events with an $\mathrm{e}^{+} \mathrm{e}^{-}$or $\mu^{+} \mu^{-}$pair and one $\tau_{\mathrm{h}}$ (right). Figures taken from [2].

The results are interpreted in various SUSY topologies. The exclusion limits at $95 \%$ confidence level in the models discussed earlier are shown in Figure 5. The existence of SUSY particles is excluded up to masses of $450-1100 \mathrm{GeV}$ depending on the SUSY scenario.
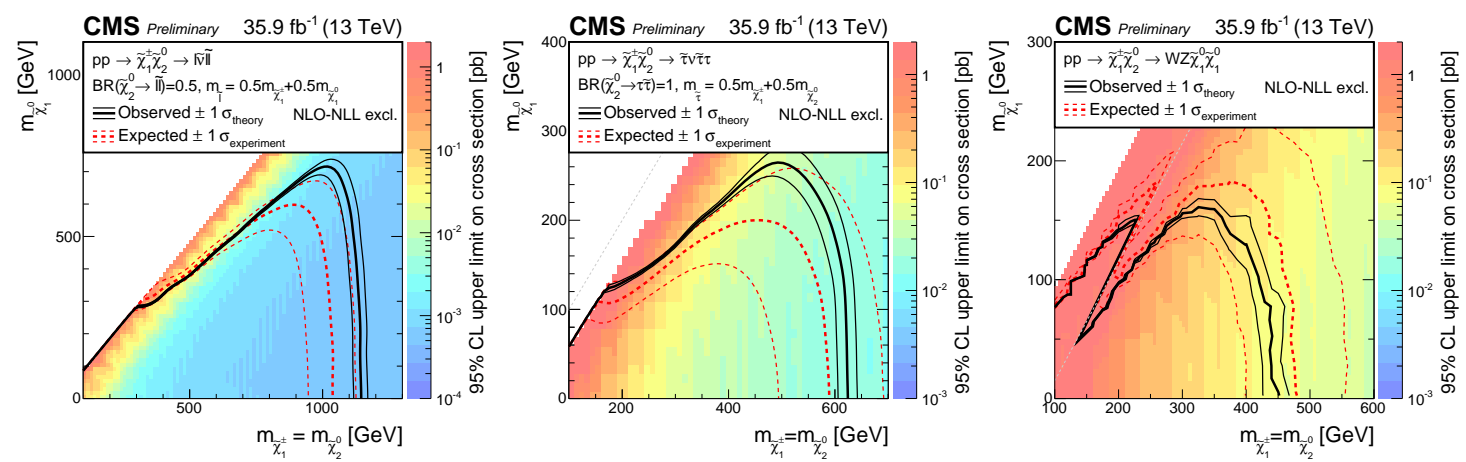

Figure 5: Interpretations of the results in the flavor-democratic light $\tilde{\ell}$ scenario (left), the tau-dominated scenario (middle), and the heavy $\tilde{\ell}$ scenario (right). The solid black lines show the observed exclusion $\left( \pm 1 \sigma_{\text {theory }}\right)$ while the red dashed lines show the expected limit $\left( \pm 1 \sigma_{\text {experiment }}\right)$. The considered SUSY scenario is excluded in the region below the respective curve. Figures taken from [2]. 


\section{References}

[1] CMS Collaboration, "The CMS Experiment at the CERN LHC", JINST 3 (2008) S08004, doi:10.1088/1748-0221/3/08/S08004.

[2] CMS Collaboration, "Search for electroweak production of charginos and neutralinos in multilepton final states in proton-proton collisions at $\sqrt{s}=13 \mathrm{TeV}$ ”, submitted to JHEP, CMS-PAS-SUS-16-039. 